\title{
Generalized Recurrent Exponential Fuzzy Associative Memories Based on Similarity Measures*
}

\author{
Aline Cristina de Souza, Marcos Eduardo Valle, and Peter Sussner \\ Department of Applied Mathematics, University of Campinas, Campinas, Brazil.
}

\begin{abstract}
The recurrent exponential fuzzy associative memory (RE-FAM) can be viewed as a recurrent neural network that employs a fuzzy similarity measure in its hidden layer. This paper introduces the generalized recurrent exponential fuzzy associative memory (GRE-FAM). In contrast to the RE-FAM, the GREFAM is equipped with a second hidden layer that is geared to avoiding crosstalk. Apart from theoretical results, this paper includes some computational experiments concerning the reconstruction of corrupted gray-scale images.
\end{abstract}

Keywords: Associative memory, recurrent neural network, fuzzy system, similarity measure.

\section{Introduction}

The (discrete) Hopfield neural network, introduced in the early 1980s, is a widely known recurrent neural network that can be used to implement an associative memory (AM) [1]. Despite having some attractive features and applications [2], the Hopfield network suffers from a low absolute storage capacity [3]. A simple but significant improvement over the Hopfield network is given by the $e x$ ponential correlation associative memory (ECAM), introduced by Chiueh and Goodman in the early 1990s [4]. Roughly speaking, Chiueh and Goodman generalized the Hopfield network by adding a layer whose nodes apply an exponential function to the correlation between the current state and a fundamental memory. The storage capacity of the ECAM scales exponentially with the length of the vectors [4]. Besides a very high storage capacity, the ECAM exhibits an excellent error correction capability but - like the discrete Hopfield network - the ECAM is only suited for storing and recalling bipolar patterns. However, many applications of AMs, including the retrieval of gray-scale images in the presence of noise, require the storage and recall of many-valued patterns such as realvalued vectors, complex-valued vectors, or fuzzy sets $[5,6,7,8,9,10,11]$.

*This work was partially supported by CAPES, FAPESP under grant no. 2013/12310-4, and CNPq under grant nos. 305486/2014-4 and 311695/2014-0.
In 1993, Chiueh and Tsai proposed an extension of the bipolar ECAM called multivalued exponential recurrent associative memory (MERAM), in order to deal with multivalued patterns [12]. The hidden nodes of the MERAM use evaluate the similarity between multivalued patterns before applying an exponential function. Then, a weighted average of the original patterns is computed.

Recently, Valle adapted the MERAM model for fuzzy sets by employing some well-known fuzzy similarity measures and by aggregating fuzzy sets using weighted averages [13]. The resulting class of models is referred to as the class of recurrent exponential fuzzy associative memories (RE-FAMs).

In some computational experiments concerning the retrieval of gray-scale images [13], RE-FAMs exhibited a better performance than other well-known AM models from the literature such as the kernel associative memory (KAM) [14], the complex-sigmoid Hopfield network [6], and a certain subspace projection autoassociative memory (SPAM) [15]. However, RE-FAM models may suffer from a problem known as "crosstalk" that is due to the interaction between the stored patterns. In this paper, we show that the crosstalk between the fundamental memories can be reduced by adding another hidden layer, which leads to a generalized recurrent exponential fuzzy associative memory (GRE-FAM), and by adjusting the parameters of this hidden layer. Furthermore, we characterize the single-step output of these GRE-FAM models when the basis of the exponential is sufficiently large. Some computational experiments concerning the retrieval of corrupted gray-scale images are also presented in this paper.

The paper is organized as follows. Section 2 briefly reviews the RE-FAM models based on similarity measures. The novel GRE-FAM models are introduced in Section 3. This section also contains some theoretical results. Some computational experiments are provided in Section 4. The paper finishes with some concluding remarks.

\section{Recurrent Exponential Fuzzy Associative Memories Based on Similarity Measures}

Let us begin by recalling some well-established basic concepts that will be used throughout the text. First of all, a fuzzy set $A$ on a universe of discourse $U$ is determined by its membership func- 
tion $A: U \rightarrow[0,1]$. Let $A(u)$ denote the degree to which the element $u \in U$ belongs to the fuzzy set $A$. The family of all fuzzy subsets of $U$ is denoted by $\mathcal{F}(U)$. Similarly, $\mathcal{P}(U)$ represents the power set of $U$. As usual, we say that $A \in \mathcal{F}(U)$ is a subset of $B \in \mathcal{F}(U)$, and write $A \subseteq B$, if $A(u) \leq B(u)$ for all $u \in U$. Moreover, $\bar{A}$ denotes the standard complement of a fuzzy set $A$, that is, $\bar{A}(u)=1-A(u), \forall u \in U$.

A similarity measure, also known as equality index or fuzzy equivalence, is a function that maps pair of fuzzy sets to a real number in the unit interval $[0,1]$, representing the degree to which those fuzzy sets are equal $[16,17]$. Applications of similarity measures include fuzzy neural networks [8, 18], fuzzy clustering [19], and rule base simplification [20]. In the following, we consider the normalized version of the axiomatic definition provided by Xuecheng [21]:

Definition 1 (Similarity Measure). A similarity measure is a function $\mathcal{S}: \mathcal{F}(U) \times \mathcal{F}(U) \rightarrow[0,1]$ which satisfies the following properties for any fuzzy sets $A, B, C, D \in \mathcal{F}(U)$ :

1. $\mathcal{S}(A, B)=S(B, A)$.

2. $\mathcal{S}(A, A)=1$.

3. If $A \subseteq B \subseteq C \subseteq D$, then $\mathcal{S}(A, D) \leq \mathcal{S}(B, C)$.

4. $\mathcal{S}(A, \bar{A})=0$, for every crisp set $A \in \mathcal{P}(U)$.

In addition, we say that $\mathcal{S}$ is a strong similarity measure if $\mathcal{S}(A, B)=1$ implies $A=B$.

Example 1. Let $U=\left\{u_{1}, \cdots, u_{n}\right\}$ be a finite universe of discourse. The following presents three instances of strong similarity measures [16, 22, 23, 24].

1. Gregson similarity measure:

$$
\mathcal{S}_{G}(A, B)=\frac{\sum_{j=1}^{n} A\left(u_{j}\right) \wedge B\left(u_{j}\right)}{\sum_{j=1}^{n} A\left(u_{j}\right) \vee B\left(u_{j}\right)},
$$

where the symbols " $\wedge$ " and " $\vee$ " denote respectively the minimum and maximum operations.

2. Eisler and Ekman similarity measure:

$$
\mathcal{S}_{E}(A, B)=\frac{2 \sum_{j=1}^{n} A\left(u_{j}\right) \wedge B\left(u_{j}\right)}{\sum_{j=1}^{n} A\left(u_{j}\right)+\sum_{j=1}^{n} B\left(u_{j}\right)} .
$$

3. Complement of a relative distance:

$$
\mathcal{S}_{p}(A, B)=1-\frac{d_{p}(A, B)}{d_{p}(\emptyset, U)},
$$

where $d_{p}$, given by the following equation, denotes the $L_{p}$ distance of order $p \geq 1$ :

$$
d_{p}(A, B)=\left(\sum_{j=1}^{n}\left|A\left(u_{j}\right)-B\left(u_{j}\right)\right|^{p}\right)^{1 / p} .
$$

The denominator $d_{p}(\emptyset, U)$, which corresponds to the largest distance between two fuzzy sets, ensures that

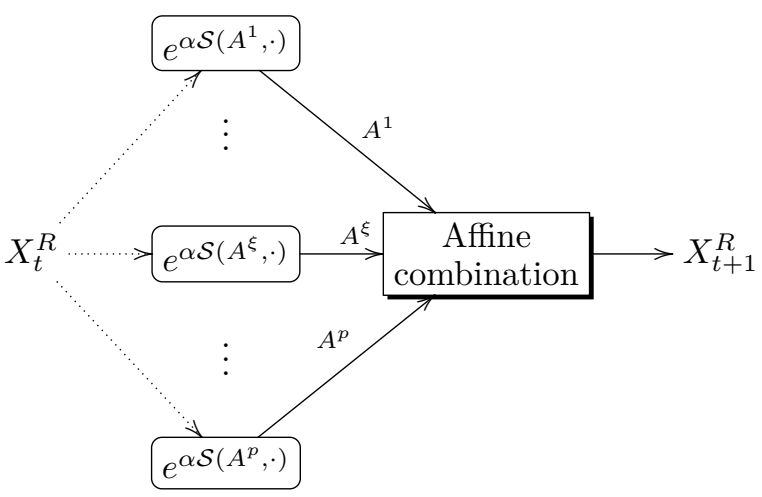

Figure 1: Block diagram of a RE-FAM.

$S_{p}(A, B) \in[0,1]$. In particular, the complement of the relative Hamming distance $\mathcal{S}_{H}$ is obtained by considering $p=1$, i.e.,

$$
\mathcal{S}_{H}(A, B)=1-\frac{1}{n} \sum_{j=1}^{n}\left|A\left(u_{j}\right)-B\left(u_{j}\right)\right| .
$$

Let us now review recurrent exponential fuzzy associative memories, also known as fuzzy exponential recurrent neural networks (FERNNs) [13]. A RE-FAM can be interpreted as a two-layer dynamic neural network designed for the storage of a finite family $\mathcal{A}=\left\{A^{1}, A^{2}, \cdots, A^{p}\right\}$ of fuzzy sets. We shall refer to $A^{\xi} \in \mathcal{A}$ as a fundamental memory. In each node of the first layer, an exponential function is applied to the similarity between $A^{\xi}$, for $\xi=1, \ldots, p$, and the current state, represented by a fuzzy set $X_{t}^{R} \in \mathcal{F}(U)$. The output layer yields an affine combination of the fundamental memories $A^{1}, \ldots, A^{p}$ whose weights are the outputs of the previous layer. Figure 1 shows a block diagram of a RE-FAM. Formally, a RE-FAM is defined as follows:

Definition 2 (RE-FAM). Consider a family of fuzzy sets $\mathcal{A}=\left\{A^{1}, \ldots, A^{p}\right\} \subseteq \mathcal{F}(U)$, a real number $\alpha>0$, and a similarity measure $\mathcal{S}: \mathcal{F}(U) \times \mathcal{F}(U) \rightarrow$ $[0,1]$. Given a fuzzy set $X_{0} \in \mathcal{F}(U)$, a $R E-F A M$ recursively produces the following sequence $\left\{X_{t}^{R}\right\}$ of fuzzy sets:

$$
X_{t+1}^{R}(u)=\frac{\sum_{\xi=1}^{p} A^{\xi}(u) e^{\alpha \mathcal{S}\left(A^{\xi}, X_{t}^{R}\right)}}{\sum_{\eta=1}^{p} e^{\alpha \mathcal{S}\left(A^{\eta}, X_{t}^{R}\right)}}, \quad \forall u \in U,
$$

where $X_{0}^{R}=X_{0}$, for all non-negative integer $t$.

Like as the correlation matrix memory [25], the RE-FAM model is subject to crosstalk between patterns as the following example illustrates.

Example 2. Consider the nine gray-scale images displayed in Figure 2. These images have size $128 \times 128$ and 256 gray levels. By dividing the 8-bit intensities by 255 , each of these images was associated with a fundamental memory $A^{\xi}: U \rightarrow[0,1]$, 

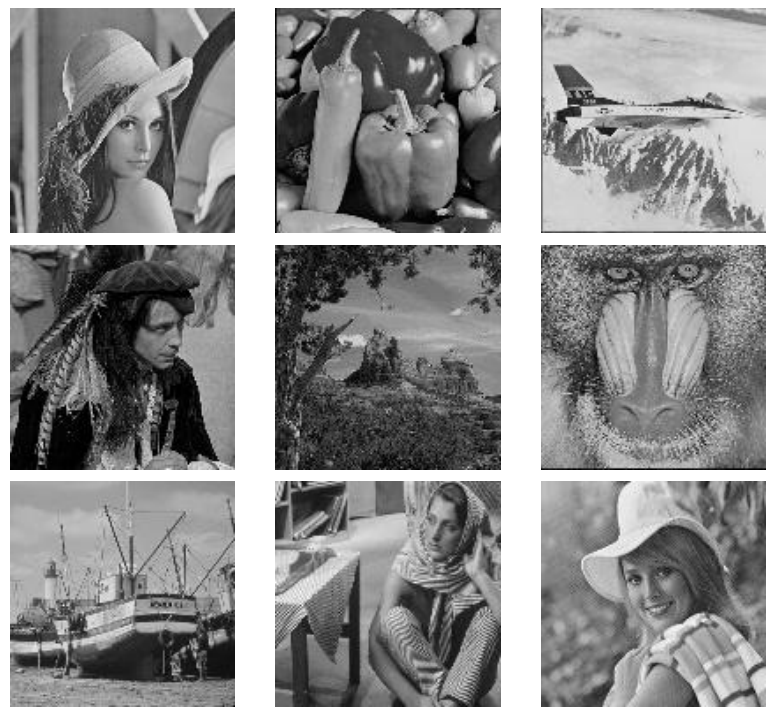

Figure 2: Original gray-scale images of size $128 \times 128$ and 256 gray-levels.
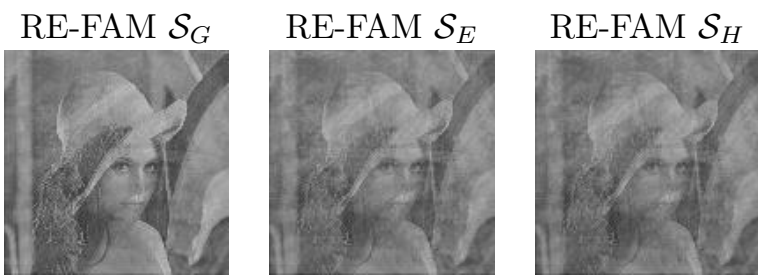

Figure 3: Images recovered by single-step RE-FAMs with $\alpha=5$ upon presentation of the original Lena image.

where $U=\{1,2, \ldots, 128\} \times\{1,2, \ldots, 128\}$. Afterwards, we presented the original Lena image to the RE-FAMs based on the similarity measures given by Example 1 and the parameter $\alpha=5$. Figure 3 shows the gray-scale images produced by the singlestep RE-FAMs. In other words, the three images depicted in Figure 3 correspond to the fuzzy set $X_{1}^{R}$ determined by (6), where $X_{0}$ represents the original Lena image, using $\alpha=5$ and the similarity measures $\mathcal{S}_{G}, \mathcal{S}_{E}$, and $\mathcal{S}_{H}$, respectively. Note that the original Lena image was not recovered by the single-step RE-FAMs due to the crosstalk between the fundamental memories $A^{1}, \ldots, A^{p}$. Specifically, if $X_{0}=A^{\mu}, \mu \in\{1, \ldots, p\}$ is fed into a RE-FAM, then (6) yields the fuzzy set

$$
X_{1}^{R}(u)=w_{\mu} A^{\mu}(u)+\sum_{\xi \neq \mu} w_{\xi} A^{\xi}(u), \quad \forall u \in U,
$$

where

$$
w_{\xi}=\frac{e^{\alpha \mathcal{S}\left(A^{\xi}, A^{\mu}\right)}}{\sum_{\eta=1}^{p} e^{\alpha \mathcal{S}\left(A^{\eta}, A^{\mu}\right)}}, \quad \forall \xi=1, \ldots, p .
$$

The term $E(u)=\sum_{\xi \neq \mu} w_{\xi} A^{\xi}(u)$ in (7) corresponds to the error due to the crosstalk.

The following theorem ${ }^{1}$ shows that the error term

\footnotetext{
${ }^{1}$ Due to restrictions in the number of pages, we postpone the proofs of some theorems to a future journal paper.
}

$E(u)$ can be made as small as desired by increasing the value of the parameter $\alpha$, that is, $E(u) \rightarrow 0$ as $\alpha \rightarrow \infty$.

Theorem 1. Consider a family of fundamental memories $\mathcal{A}=\left\{A^{1}, \cdots, A^{p}\right\} \subseteq \mathcal{F}(U)$ and a similarity measure $\mathcal{S}$. Given a fuzzy set $X_{0} \in \mathcal{F}(U)$, let $\Gamma \subseteq\{1, \ldots, p\}$ denote the set of the indexes of the fundamental memories which are the most similar to the input $X_{0}$ in terms of $\mathcal{S}$. Formally, we have

$$
\Gamma=\left\{\gamma: \mathcal{S}\left(A^{\gamma}, X_{0}\right) \geq \mathcal{S}\left(A^{\xi}, X_{0}\right), \forall \xi=1, \ldots, p\right\} .
$$

If $X_{1}^{R} \in \mathcal{F}(U)$, given by (6) with $t=0$, denotes the output of the single-step RE-FAM, then

$$
\lim _{\alpha \rightarrow \infty} X_{1}^{R}(u)=\frac{1}{\operatorname{Card}(\Gamma)} \sum_{\gamma \in \Gamma} A^{\gamma}(u), \quad \forall u \in U .
$$

Accordingly, by increasing the parameter $\alpha$ from 5 to 20 , the three single-step RE-FAMs considered in Example 2 produced images that are visually indistinguishable from the original Lena image. Furthermore, the RE-FAM based on the similarity measure $\mathcal{S}_{G}$ with $\alpha=20$ exhibited excellent tolerance with respect to noise in some experiments concerning the reconstruction of corrupted gray-scale images [13]. In practice, however, the implementation of a RE-FAM with large $\alpha$ may be limited by the floating point representation of real numbers. The generalized RE-FAM introduced in the following section can mitigate the adverse effects of the crosstalk between the fundamental memories independently of the value of the parameter $\alpha$.

\section{Generalized Recurrent Exponential Fuzzy Associative Memories}

Let us now generalize the RE-FAM model by adding a hidden layer before computing the affine combination. As in the original RE-FAM, the first layer nodes of a generalized RE-FAM (GRE-FAM) applies an exponential function to the similarity between $A^{\xi}$ and the current state $X_{t}^{G}$, for $\xi=1, \ldots, p$. The second hidden layer consists of linear neurons. Finally, the output layer computes an affine combination of the fundamental memories $A^{1}, \ldots, A^{p}$ followed by a piece-wise linear function $\varphi$ which ensures that $X_{t+1}^{G}(u) \in[0,1]$ for all $u \in U$. A block diagram of a GRE-FAM is shown in Figure 4. In mathematical terms, we have the following definition:

Definition 3 (GRE-FAM). Consider a family of fundamental memories $\mathcal{A}=\left\{A^{1}, A^{2}, \cdots, A^{p}\right\} \subseteq$ $\mathcal{F}(U)$, a real number $\alpha>0$, and a similarity measure $\mathcal{S}: \mathcal{F}(U) \times \mathcal{F}(U) \rightarrow[0,1]$. Furthermore, let $G=\left(g_{\nu \mu}\right)$ be a real-valued matrix of size $p \times p$. Given an initial fuzzy set $X_{0} \in \mathcal{F}(U)$, a GRE-FAM 


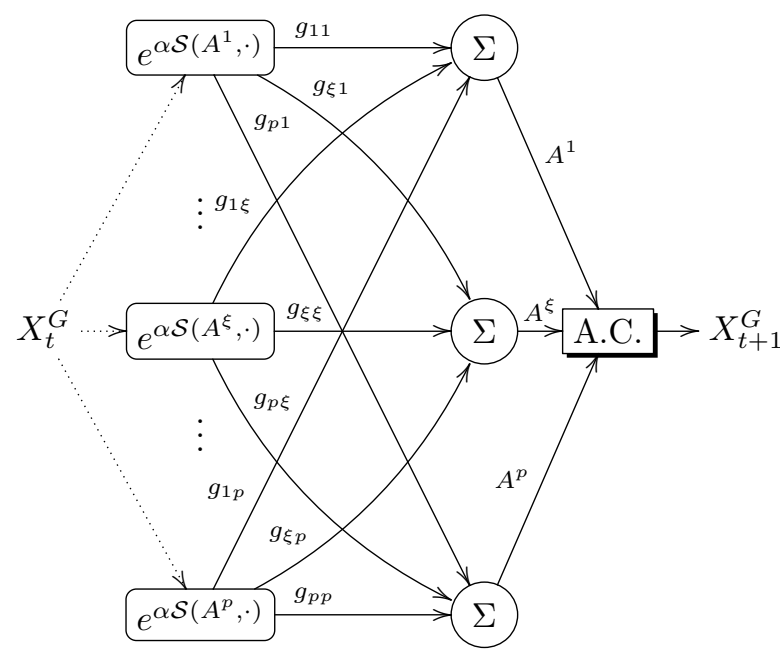

Figure 4: Block diagram of a GRE-FAM.

defines recursively the following fuzzy sets for all $u \in U$ and $t=0,1, \ldots$, where $X_{0}^{G}=X_{0}$ :

$$
X_{t+1}^{G}(u)=\varphi\left(\frac{\sum_{\xi=1}^{p} \sum_{\mu=1}^{p} A^{\xi}(u) g_{\xi \mu} e^{\alpha \mathcal{S}\left(A^{\mu}, X_{t}^{G}\right)}}{\sum_{\eta=1}^{p} \sum_{\mu=1}^{p} g_{\eta \mu} e^{\alpha \mathcal{S}\left(A^{\mu}, X_{t}^{G}\right)}}\right),
$$

where $\varphi: \mathbb{R} \rightarrow[0,1]$ is the piece-wise linear function given by

$$
\varphi(x)= \begin{cases}0, & x<0 \\ x, & 0 \leq x \leq 1, \\ 1, & x>1\end{cases}
$$

Note that the GRE-FAM model described by (11) generalizes the RE-FAM model given by (6). In fact, we obtain a RE-FAM from (11) by setting $G$ equal to the identity matrix, i.e., $G=I_{p \times p}$. Moreover, the following theorem determines a matrix $G$ such that any fundamental memory $A^{\xi}$ is a fixed point of (11).

Theorem 2. Given a family of fundamental memories $\mathcal{A}=\left\{A^{1}, A^{2}, \cdots, A^{p}\right\} \subseteq \mathcal{F}(U)$, a parameter $\alpha>0$, and a similarity measure $\mathcal{S}: \mathcal{F}(U) \times \mathcal{F}(U) \rightarrow$ $[0,1]$, let the matrix $C=\left(c_{\nu \mu}\right) \in \mathbb{R}^{p \times p}$ be defined as follows:

$$
c_{\nu \mu}=e^{\alpha \mathcal{S}\left(A^{\nu}, A^{\mu}\right)}, \quad \forall \nu, \mu=1, \cdots, p .
$$

If $C$ is invertible, then any fundamental memory from the family $\mathcal{A}$ is a fixed point of the GRE-FAM with $G=C^{-1}$.

Example 3. Let $A^{1}, \ldots, A^{9}$ be the fundamental memories that correspond to the nine gray-scale images shown in Figure 2. The matrix $C$ given by (13) with $\alpha=5$ and either $\mathcal{S}_{G}, \mathcal{S}_{E}$, or $\mathcal{S}_{H}$ is invertible. Furthermore, all fundamental memories - including the original Lena image - are fixed points of the GRE-FAM with $G=C^{-1}$. In other words, if $X_{0}=A^{\mu}, \mu \in\{1, \ldots, p\}$, then (11) yields $X_{1}=A^{\mu}$.

Let us now turn our attention to the output $X_{1}^{G}$ produced by a single-step GRE-FAM with $G$ prescribed by Theorem 2. Specifically, the following theorem shows that $X_{1}^{G}$ converges point-wise to an affine combination of the fundamental memories which have the highest similarity (in terms of $\mathcal{S}$ ) with the input $X_{0}$ as the parameter $\alpha>0$ tends to infinity.

Theorem 3. Consider a family of fundamental memories $\mathcal{A}=\left\{A^{1}, \cdots, A^{p}\right\} \subseteq \mathcal{F}(U)$ and let $\mathcal{S}$ denote a strong similarity measure. Suppose that the matrix $C$ given by (13) is invertible for any $\alpha>0$. If the single-step GRE-FAM with $G=C^{-1}$ produces the output $X_{1}^{G} \in \mathcal{F}(U)$ upon presentation of $X_{0} \in \mathcal{F}(U)$, then

$$
\lim _{\alpha \rightarrow \infty} X_{1}^{G}(u)=\frac{1}{\operatorname{Card}(\Gamma)} \sum_{\gamma \in \Gamma} A^{\gamma}(u), \quad \forall u \in U,
$$

where $\Gamma$ is the set of indexes given by (9).

Proof. Let $\sigma=\max _{\xi=1: p}\left\{\mathcal{S}\left(A^{\xi}, X_{0}\right)\right\}$ denote the maximum of the similarity between the input fuzzy set $X_{0}$ and the fundamental memories $A^{1}, \cdots, A^{p}$. The output of a single-step GRE-FAM satisfies

$$
X_{1}^{G}(u)=\varphi\left(\sum_{\xi=1}^{p} w_{\xi} A^{\xi}(u)\right)
$$

where $w_{\xi}$, for $\xi=1, \ldots, p$, is given by

$$
w_{\xi}=\frac{\sum_{\mu=1}^{p} g_{\xi \mu} e^{\alpha \mathcal{S}\left(A^{\mu}, X_{0}\right)}}{\sum_{\eta=1}^{p} \sum_{\mu=1}^{p} g_{\eta \mu} e^{\alpha \mathcal{S}\left(A^{\mu}, X_{0}\right)}} .
$$

Multiplying both the numerator and the denominator of (16) by $e^{-\alpha \sigma}$ and breaking up the sums, we obtain:

$$
w_{\xi}=\frac{\sum_{\gamma \in \Gamma} g_{\xi \gamma}+\sum_{\mu \notin \Gamma} g_{\xi \mu} e^{\alpha\left(\mathcal{S}\left(A^{\mu}, X_{0}\right)-\sigma\right)}}{\sum_{\eta=1}^{p} \sum_{\gamma \in \Gamma} g_{\eta \gamma}+\sum_{\eta=1}^{p} \sum_{\mu \notin \Gamma} g_{\eta \mu} e^{\alpha\left(\mathcal{S}\left(A^{\mu}, X_{0}\right)-\sigma\right)}} .
$$

Now, the matrix $C$ given by (13) can be written as $C=e^{\alpha} D(\alpha)$, where the entries of $D(\alpha)$ are $d_{\nu \mu}(\alpha)=e^{\alpha\left(\mathcal{S}\left(A^{\nu}, A^{\mu}\right)-1\right)}$. Moreover, $G=C^{-1}=$ $e^{-\alpha} H(\alpha)$, where $H(\alpha)=D^{-1}(\alpha)$. Hence, by factoring $e^{-\alpha}$, we obtain from (17):

$$
w_{\xi}=\frac{\sum_{\gamma \in \Gamma} h_{\xi \gamma}(\alpha)+\sum_{\mu \notin \Gamma} h_{\xi \mu}(\alpha) e^{\alpha\left(\mathcal{S}\left(A^{\mu}, X_{0}\right)-\sigma\right)}}{\sum_{\eta=1}^{p} \sum_{\gamma \in \Gamma} h_{\eta \gamma}(\alpha)+\sum_{\eta=1}^{p} \sum_{\mu \notin \Gamma} h_{\eta \mu}(\alpha) e^{\alpha\left(\mathcal{S}\left(A^{\mu}, X_{0}\right)-\sigma\right)}} .
$$

Recalling that $\mathcal{S}\left(A^{\mu}, X_{0}\right)-\sigma<0$ for all $\mu \notin \Gamma$, the second sum in both numerator and denominator tends to 0 as $\alpha \rightarrow \infty$. Moreover, since $\mathcal{S}$ 


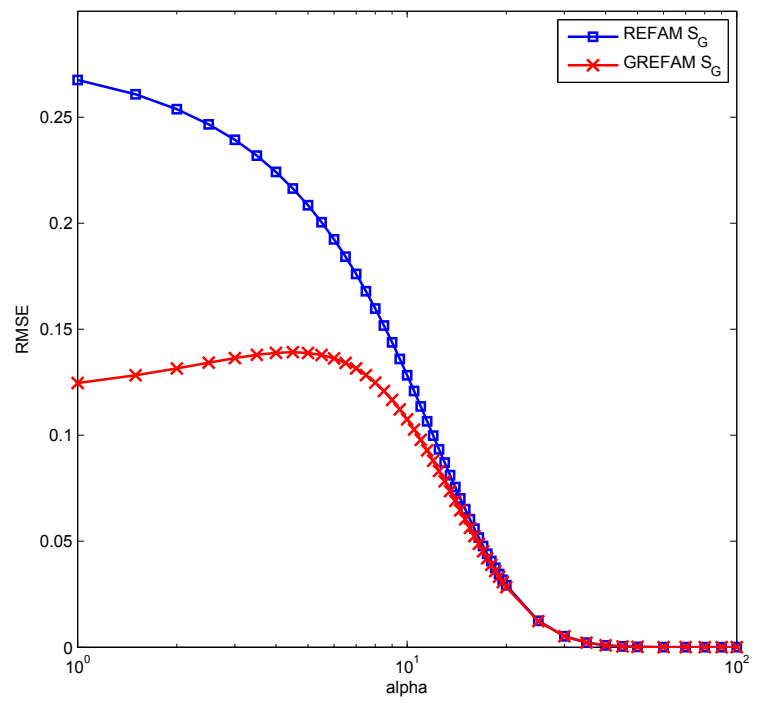

Figure 5: RMSE between $A^{3}$ and the fuzzy set retrieved by a single-step model by the parameter $\alpha$.

is a strong similarity measure, $\lim _{\alpha \rightarrow \infty} h_{\nu \mu}(\alpha)=\delta_{\nu \mu}$, where $h_{\nu \mu}(\alpha)$ is the $(\nu, \mu)$-entry of $H(\alpha)$ and $\delta_{\nu \mu}$ denotes the Kronecker's delta. Hence,

$$
\begin{aligned}
& \lim _{\alpha \rightarrow \infty} X_{1}(u)=\lim _{\alpha \rightarrow \infty} \varphi\left(\sum_{\xi=1}^{p} w_{\xi} A^{\xi}(u)\right) \\
& =\varphi\left(\frac{\sum_{\xi=1}^{p} \sum_{\gamma \in \Gamma} \delta_{\xi \gamma} A^{\xi}(u)}{\sum_{\eta=1}^{p} \sum_{\gamma \in \Gamma} \delta_{\eta \gamma}}\right)=\frac{\sum_{\gamma \in \Gamma} A^{\gamma}(u)}{\operatorname{Card}(\Gamma)},
\end{aligned}
$$

for all $u \in U$.

By comparing (10) and (14), we conclude that the single-step RE-FAM and GRE-FAM - both based on the same similarity measure and fundamental memories - yield the same fuzzy set upon the presentation of $X_{0}$ for $\alpha$ sufficiently large. The following example illustrates this remark.

Example 4. Consider the fundamental memories $A^{1}, \ldots, A^{9}$ corresponding to the nine gray-scale images displayed in Figure 2 and let $X_{0} \in \mathcal{F}(U)$ be the fuzzy set given by $X_{0}(u)=1$ for all $u \in U$. Visually, $X_{0}$ corresponds to a white image. By considering the similarity measure $\mathcal{S}_{G}$ given by (1), we obtain the similarity degrees shown in Table 1 . Note that $A^{3}$ is the fundamental memory that is most similar to the input $X_{0}$ in terms of $S_{G}$ and, consequently, $\Gamma=\{3\}$. Now, let $X_{1}^{R}$ and $X_{1}^{G}$ denote respectively the fuzzy sets retrieved by the single-step RE-FAM and the GRE-FAM with $G=C^{-1}$ under presentation of $X_{0}$. Figure 5 shows the root-mean-squared error (RMSE) produced by $X_{1}^{R}$ and $X_{1}^{G}$ by the parameter $\alpha$. We would like to remark that the RMSE is given by the relative Euclidean distance between the desired fuzzy set $A^{\xi}, \quad x i \in\{1, \ldots, p\}$, and the output $Y \in \mathcal{F}(U)$ yielded by a model, i.e.,

$$
\operatorname{RMSE}\left(A^{\xi}, Y\right)=\sqrt{\frac{\sum_{u \in U}\left(A^{\xi}(u)-Y(u)\right)^{2}}{\operatorname{Card}(U)}} .
$$

As expected, $\operatorname{RMSE}\left(A^{3}, X_{1}^{R}\right)$ as well as $\operatorname{RMSE}\left(A^{3}, X_{1}^{G}\right)$ approaches zero as $\alpha$ tends to infinity. In fact, we have $\operatorname{RMSE}\left(A^{3}, X_{1}^{R}\right) \leq 10^{-6}$ and $\operatorname{RMSE}\left(A^{3}, X_{1}^{G}\right) \leq 10^{-6}$ for any $\alpha \geq 90$. In other words, both $X_{1}^{R} \rightarrow A^{3}$ and $X_{1}^{G} \rightarrow A^{3}$ for $\alpha \rightarrow \infty$ in accordance with Theorems 1 and 3 .

Remark 1. We obtained similar results by considering the other two similarity measures given in Example 1. In particular, both $\mathcal{S}_{E}$ and $\mathcal{S}_{H}$ yielded $\Gamma=\{3\}$ because $A^{3}$ corresponds to the whiter image - thus, the most similar to $X_{0}$ - among the nine gray-scale images depicted in Figure 2.

Concluding, given fundamental memories $A^{1}, \ldots, A^{p}$ and a strong similarity measure $\mathcal{S}$, the single-step RE-FAM and the GRE-FAM with $G=C^{-1}$ yield almost the same output when the parameter $\alpha$ tends to infinity. Computationally, the design of a GRE-FAM based on Theorem 2 requires $\mathcal{O}\left(p^{3}\right)$ operations. Apart from evaluating the piece-wise linear function $\varphi$ given by (12), a GRE-FAM performs $\mathcal{O}\left(p^{2}\right)$ more operations per step than a RE-FAM due to the additional layer.

In many practical situations, however, the parameter $\alpha$ cannot be made indefinitely large. For instance, due to overflow, we must roughly consider $\alpha \leq 700$ on a machine that supports IEEE floating point arithmetic. If the parameter $\alpha$ is not sufficiently large, the fuzzy sets produced by the REFAM and the GRE-FAM may differ significantly. Examples 2 and 3, where the RE-FAM failed but the GRE-FAM succeed to retrieve the original Lena image, illustrate this remark. In fact, from Theorem 2 , the fundamental memories $A^{1}, \ldots, A^{p}$ are all fixed points of the GRE-FAM with $G=C^{-1}$. In other words, this memory model has optimal absolute storage capacity. The computational experiments provided in the following section reveal that this GRE-FAM also exhibits an excellent noise tolerance even if $\alpha$ is not very large. In particular, we shall see that the GRE-FAM with $G=C^{-1}$ outperforms the RE-FAM in experiments concerning the reconstruction of corrupted gray-scale images.

\section{Computational Experiments}

Let $A^{1}, \ldots, A^{9}$ be the fuzzy sets corresponding to the nine gray-scale images displayed in Figure 2. First, we synthesized a RE-FAM and a GRE-FAM model using the family of fundamental memories $\mathcal{A}=\left\{A^{1}, \ldots, A^{9}\right\}$, the parameter $\alpha=20$, and the similarity measures $\mathcal{S}_{G}, \mathcal{S}_{E}$, and $\mathcal{S}_{H}$ defined respectively by (1), (2), and (5). As suggested by Theorem 2 , the matrix of the GRE-FAM is determined by the equation $G=C^{-1}$, where $C$ is given by (13). 


\begin{tabular}{c|c|c|c|c|c|c|c|c}
$\mathcal{S}_{G}\left(A^{1}, x_{0}\right)$ & $\mathcal{s}_{G}\left(A^{2}, x_{0}\right)$ & $\mathcal{S}_{G}\left(A^{3}, x_{0}\right)$ & $\mathcal{s}_{G}\left(A^{4}, x_{0}\right)$ & $\mathcal{s}_{G}\left(A^{5}, x_{0}\right)$ & $\mathcal{S}_{G}\left(A^{6}, x_{0}\right)$ & $\mathcal{S}_{G}\left(A^{7}, x_{0}\right)$ & $\mathcal{s}_{G}\left(A^{8}, x_{0}\right)$ & $\mathcal{S}_{G}\left(A^{9}, x_{0}\right)$ \\
\hline 0.4865 & 0.4061 & $\mathbf{0 . 6 9 9 0}$ & 0.3080 & 0.3396 & 0.5028 & 0.5337 & 0.4607 & 0.5348
\end{tabular}

Table 1: Degree of similarity between the input fuzz set $X_{0}$ and the fundamental memories $A^{1}, \ldots, A^{9}$ of Example 4.

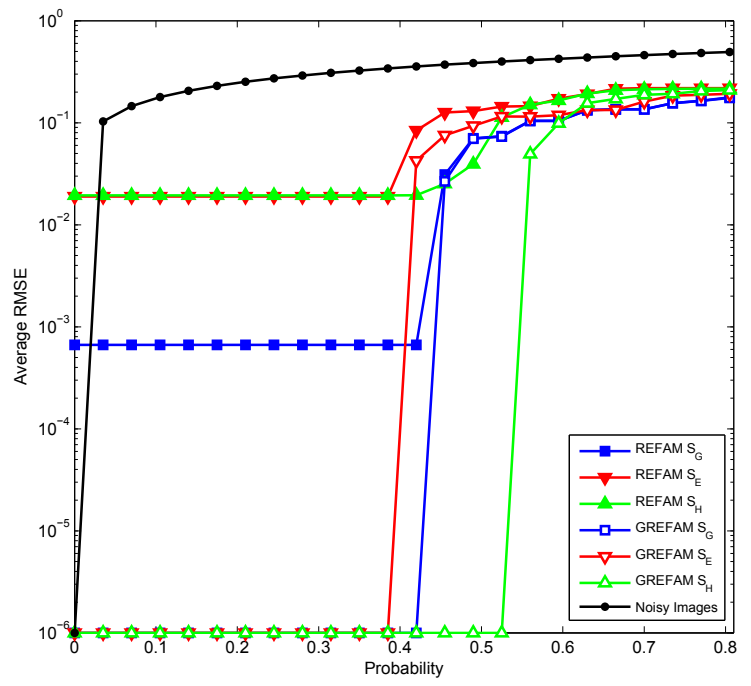

Figure 6: Average RMSE by the probability of salt and pepper noise added to the input image.

In the computational experiments, the outputs of the RE-FAM and GRE-FAM have been obtained by iterating respectively (6) and (11) until either $d_{2}\left(X_{t}, X_{t+1}\right) / d_{2}(\emptyset, U) \leq 10^{-6}$ or $t>20$.

We probed each model with images corrupted by salt and pepper noise with densities varying from 0 to 0.8 . We also probed the AM models with images corrupted by Gaussian noise with zero mean and variance ranging from 0 to 0.15 as well as blurred images obtained by an approximation of the horizontal motion of a camera by $h \in\{1,3,5, \ldots, 51\}$ pixels. Figures 6,7 , and 8 compares the average RMSEs produced by the six models averaged in 90 experiments, that is, each original image was distorted 10 times for a certain noise or blur intensity. The average RMSEs produced by the corrupted images are also shown in these figures.

First of all, note that the average RMSE produced by a GRE-FAM is at most as large as the average RMSE yielded by its corresponding RE-FAM model. In particular, observe in Figure 6 that the GRE-FAM based on $\mathcal{S}_{H}$ exhibited the best tolerance with respect to salt and pepper noise with probability $p$ less than or equal to 0.525 . All the models produced similar average RMSEs for images corrupted by salt and pepper noise with $p \geq 0.6$. Similarly, Figures 7 and 8 reveal that the GRE-FAM based on Gregson's similarity measure $\mathcal{S}_{G}$ exhibited the best tolerance with respect to both Gaussian noise with variance $\sigma^{2} \leq 0.075$ and blurred im-

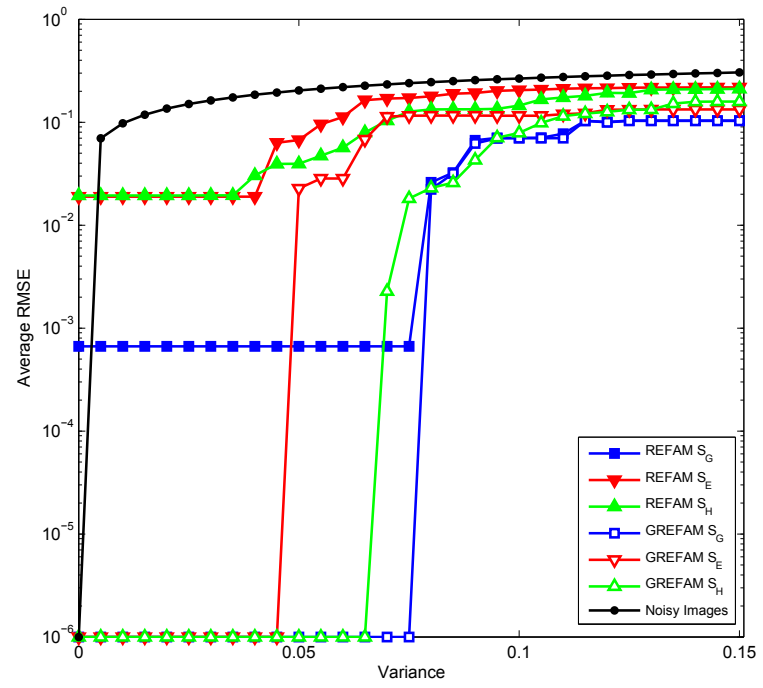

Figure 7: Average RMSE by the variance of Gaussian noise added to the input image

ages obtained by an horizontal motion of $h \leq 33$ pixels. The GRE-FAM and the RE-FAM based on $\mathcal{S}_{G}$ yielded almost the same RMSEs for images corrupted by Gaussian noise with $\sigma^{2} \geq 0.08$ or blurred images with $h \geq 35$.

Finally, Figures 9, 10, and 11 provide visual examples of the corrupted input image and the corresponding images retrieved by the AM models. Precisely, Figure 9 shows the image "peppers" corrupted by salt and pepper noise with probability $p=0.45$ followed by the images retrieved by the RE-FAM and GRE-FAM based on the similarity measures $\mathcal{S}_{G}, \mathcal{S}_{E}$, and $\mathcal{S}_{H}$. Note that both REFAM and GRE-FAM based on $\mathcal{S}_{E}$ failed to retrieve the original gray-scale image. Similarly, Figure 10 shows the image "peppers" corrupted by Gaussian noise with zero mean and variance $\sigma^{2}=0.07$ followed by images retrieved by the AM models. The RE-FAM based on $\mathcal{S}_{H}$ as well as the RE-FAM and GRE-FAM based on $\mathcal{S}_{E}$ failed to retrieve the original image. Also, Figure 11 depicts a blurred image obtained by a horizontal motion of $h=20$ pixels and the corresponding recalled images. In this case, the RE-FAMs based on $\mathcal{S}_{E}$ and $\mathcal{S}_{H}$ failed to retrieve the original "peppers" image. Finally, although the RE-FAM models retrieved images visually similar to the original "peppers" image, their RMSEs are often much larger than the RMSEs produced by the GRE-FAMs. 


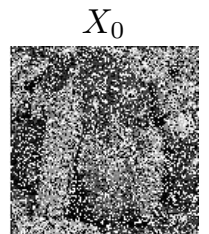

0.3765

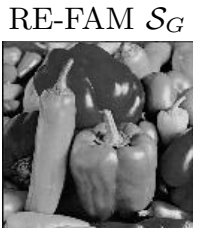

$2.4626 \times 10^{-4}$

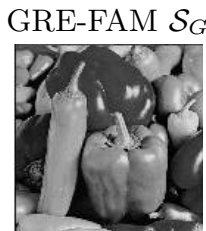

$3.3317 \times 10^{-9}$

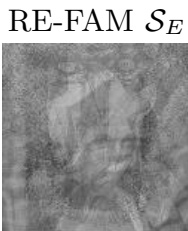

0.2582

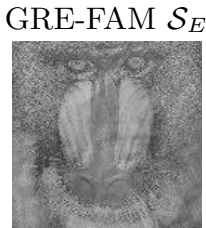

0.2604

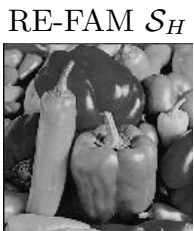

0.0159

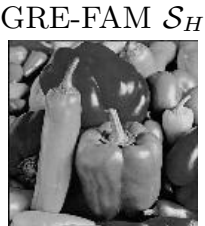

$3.7889 \times 10^{-7}$

Figure 9: Input image corrupted by salt and pepper noise with probability 0.45 followed by the images retrieved by the RE-FAM and GRE-FAM models. The last row provides the corresponding RMSE.

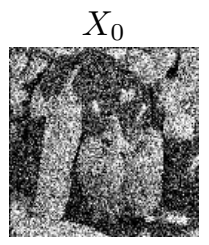

0.2285

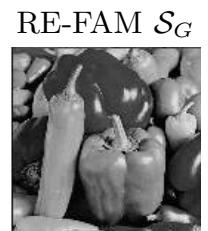

$2.4626 \times 10^{-4}$

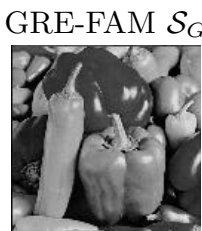

$2.0323 \times 10^{-9}$

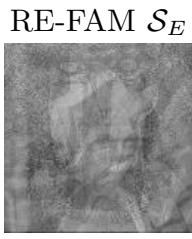

0.2582

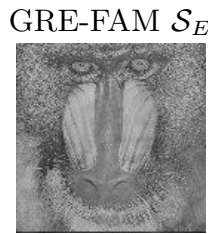

0.2656

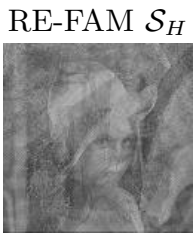

0.2474

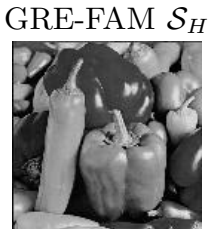

$2.8161 \times 10^{-7}$

Figure 10: Input image corrupted by Gaussian noise with zero mean and variance 0.07 followed by the images retrieved by the RE-FAM and GRE-FAM models. The last row shows the corresponding RMSE.

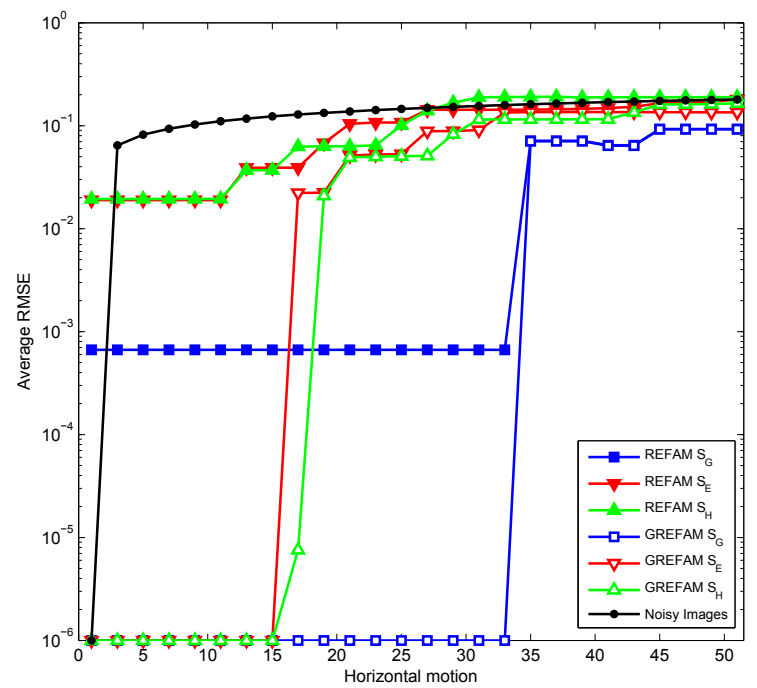

Figure 8: Average RMSE by the number of pixels of an horizontal motion of the camera.

\section{Concluding Remarks}

In this paper, we introduced the generalized recurrent exponential fuzzy associative memories (GREFAM) that can implement high-capacity associative memories for storing and recalling fuzzy sets $A^{1}, \ldots, A^{p}$. In few words, the novel models are obtained from the previous recurrent exponential fuzzy associative memories (RE-FAM) by adding a layer of traditional linear artificial neurons. Also, as pointed out by Theorem 2, this hidden layer can be used to mitigate the crosstalk between the fundamental memories $A^{1}, \ldots, A^{p}$. In addition, we provided a theorem that characterizes the output of a broad class of single-step GRE-FAMs (cf. Theorem 3 ). Roughly speaking, for a sufficiently large parameter $\alpha>0$, a GRE-FAM yields an average of the fundamental memories which are the most similar to the input. Finally, computational experiments concerning the reconstruction of noisy grayscale images revealed that the novel models, besides the optimal absolute storage capacity, may exhibit an excellent noise tolerance.

In the future, we plan to investigate further the effect of the similarity measure on the storage capacity as well as the noise tolerance of a GRE-FAM. The relationship between the novel memories and other fuzzy AM models, including the bank of fuzzy associative memories of Kosko [11, 26] and the similarity measure fuzzy associative memory of Esmi and Sussner [8, 7], require further attention. Finally, since an fuzzy associative memory can be used as a fuzzy inference engine [26], applications of the GRE-FAM in rule-based systems can be explored in the future.

\section{References}

[1] J. Hopfield. Neural Networks and Physical Systems with Emergent Collective Computational Abilities. Proceedings of the National Academy of Sciences, 79:2554-2558, April 1982.

[2] J. Hopfield and D.W. Tank. Neural computation of decisions in optimization problems. Biological Cybernetics, 52:141-152, 1985.

[3] R. McEliece, E. Posner, E. Rodemich, and S. Venkatesh. The capacity of the Hopfield associative memory. IEEE Transactions on Information Theory, 1:33-45, 1987.

[4] T. Chiueh and R. Goodman. Recurrent Correlation Associative Memories. IEEE Trans. on Neural Networks, 2:275-284, February 1991.

[5] D. Zhang and W. Zuo. Computational Intelligence-Based Biometric Technologies. 


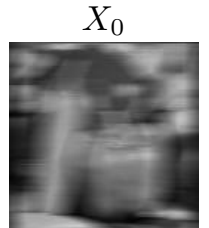

0.1509

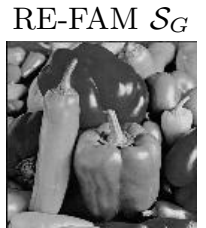

$2.4626 \times 10^{-4}$

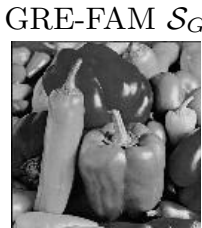

$3.2865 \times 10^{-10}$

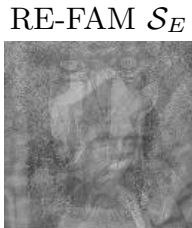

0.2582

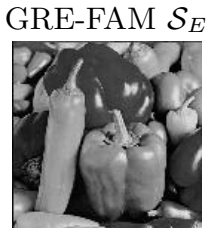

$1.6674 \times 10^{-7}$

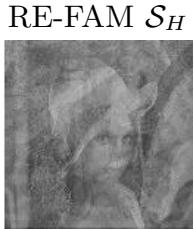

0.2481

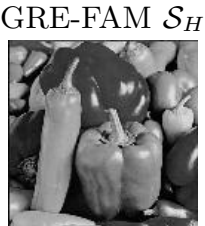

$1.7493 \times 10^{-7}$

Figure 11: Blurred input image obtained by an horizontal motion of 20 pixels followed by the images retrieved by the RE-FAM and GRE-FAM models. The last row contains the corresponding RMSE.

IEEE Computational Intelligence Magazine, 2(2):26-36, May 2007.

[6] G. Tanaka and K. Aihara. Complex-Valued Multistate Associative Memory With Nonlinear Multilevel Functions for Gray-Level Image Reconstruction. IEEE Transactions on Neural Networks, 20(9):1463-1473, September 2009.

[7] E. Esmi, P. Sussner, M. Valle, F. Sakuray, and L. Barros. Fuzzy Associative Memories Based on Subsethood and Similarity Measures with Applications to Speaker Identification. In Lecture Notes in Computer Science: International Conference on Hybrid Artificial Intelligence Systems (HAIS 2012), pages 479-490. Springer-Verlag Berlin Heidelberg, Berlin, Germany, 2012.

[8] E. Esmi, P. Sussner, H. Bustince Sola, and J.Fernandez. $\Theta$-fuzzy associative memories $(\Theta-$ FAMs). IEEE Transactions on Fuzzy Systems, 23(2):313 - 326, 2015.

[9] P. Sussner, E. Esmi, I. Villaverde, and M. Graña. The Kosko Subsethood Fuzzy Associative Memory (KS-FAM): Mathematical Background and Applications in Computer Vision. Journal of Mathematical Imaging and Vision, 42(2-3):134-149, February 2012.

[10] P. Sussner, R. Miyasaki, and M. Valle. An Introduction to Parameterized IFAM Models with Applications in Prediction. In Proceedings of the 2009 IFSA World Congress and 2009 EUSFLAT Conference, pages 247-252, Lisbon, Portugal, July 2009.

[11] S. Kong and B. Kosko. Adaptive Fuzzy Systems for Backing up a Truck-and-Trailer. IEEE Transactions on Neural Networks, 3(2):211223, 1992.

[12] T. Chiueh and H. Tsai. Multivalued associative memories based on recurrent networks. IEEE Transactions on Neural Networks, 4(2):364366, March 1993.

[13] M. Valle. Fuzzy Exponential Recurrent Neural Networks for Gray-scale Image Retrieval. In Proceedings of the Third Brazilian Conference on Fuzzy Systems (III CBSF), João Pessoa, Brazil, August 2014.

[14] B. Zhang, H. Zhang, and S. Ge. Face Recognition by Applying Wavelet Subband Representation and Kernel Associative Memory. IEEE Transactions on Neural Networks, 15(1):166-
177, January 2004.

[15] M. Valle. A Robust Subspace Projection Autoassociative Memory Based on the M-Estimation Method. IEEE Transactions on Neural Networks and Learning Systems, 25(7):1372-1377, July 2014.

[16] D. Dubois and H. Prade. Fuzzy sets and systems: theory and applications. Academic Press, New York, 1980.

[17] G. Klir and B. Yuan. Fuzzy Sets and Fuzzy Logic: Theory and Applications. Prentice Hall, Upper Saddle River, NY, 1995.

[18] R. Ballini and F. Gomide. Equality index and learning in recurrent fuzzy neural networks. In Proceedings of the 12th IEEE International Conference on Fuzzy Systems (FUZZIEEE 2003)., volume 1, pages $155-160$, St Louis, USA, May 2003.

[19] R. Yager and D. Filev. Summarizing data using a similarity based mountain method. Information Sciences, 178(3):816-826, February 2008.

[20] M. Setnes, R. Babuska, U. Kaymak, and H. van Nauta Lemke. Similarity measures in fuzzy rule base simplification. Systems, Man, and Cybernetics, Part B: Cybernetics, IEEE Transactions on, 28(3), Jun 1998.

[21] L. Xuecheng. Entropy, distance measure and similarity measure of fuzzy sets and their relations. Fuzzy Sets and Systems, 52(3):305-318, 1992.

[22] R. Zwick, E. Caristein, and D. Budescu. Measures of Similarity Among Fuzzy Concepts: A Comparative Analysis. International Journal of Approximate Reasoning, 1(2):221-242, April 1987.

[23] R. Lowen and W. Peeters. Distances between fuzzy sets representing grey level images. Fuzzy Sets and Systems, 99(2):135 - 149, 1998.

[24] I. Bloch. On fuzzy distances and their use in image processing under imprecision. Pattern Recognition, 32(11):1873 - 1895, 1999.

[25] S. Haykin. Neural Networks and Learning $M a-$ chines. Prentice-Hall, Upper Saddle River, NJ, 3rd edition edition, 2009.

[26] B. Kosko. Neural Networks and Fuzzy Systems: A Dynamical Systems Approach to Machine Intelligence. Prentice Hall, Englewood Cliffs, NJ, 1992. 